Postgrad. med. J. (September 1968) 44, 667.

\title{
Discussion to the paper by B. C. Morson
}

\section{Chatrman: Professor J. C. Goligher}

Chairman. Could Dr Morson tell us exactly what vascular abnormalities were found in the vessels in his particular specimens? I wasn't sure whether he had found many of these. What did he actually find in his specimens?

Morson. The answer to the question is 'no'. I've found that there have been no vascular abnormalities in the blood vessels that have been made available to me by the surgeon. But I think that there is radiological evidence, particularly through selective mesenteric arteriography, that in some of these cases there is a block, or there may be a block, that has not been given. Here I am trespassing on the ground of my colleague Adrian Marston who knows much more about this than I do, and I think he is rather keen on the idea that there is a sort of reflex shut-down of peripheral vessels that contributes as much to the ischaemia as to any mechanical blockage of a major vessel. Now, I dare say there are exceptions to what I have said, but not within my experience. There is a very interesting paper from Professor Louw's unit, in Capetown, published in the British Journal of Radiology $(1966,39,575)$ in which cases of ischaemic colitis are described which are undoubtedly due to atheromatous embolism to peripheral blood vessels of the colon, beautifully illustrated with microphotographs. But I have never seen this in any of my specimens.

ChaIRMan. Have selective arteriographic studies been pursued energetically, do you know what has actually been found?

MoRson. I've only read about them, and I know they are being done at the Middlesex Hospital. Adrian Marston, I understand, has produced acute ischaemic colitis, transient ischaemic colitis and ischaemic stricture in dogs experimentally. This has also been successfully produced in Professor Louw's unit at Capetown, and it particularly pleased me to look at the section from the Middlesex Hospital and in Professor Louw's reports which show that the histopathology of the lesion in the dog is exactly like one sees in the human being. I think this work on animal models of human ischaemic disease of the colon is a very satisfying example of how the animal can be useful in confirming perhaps what we have suspected to be the position.

WALKER. We've seen several of these cases as well as the radiological sign, so called 'thumb-printing'. Will these correspond to these saccules that you described?

Morson. I've had some arguments with my radiology colleagues about this. I think they must do, but they rather give me the impression that they think that the saccules are sort of 'inside-out' from their point of view. What I think happens is that this could be disease in the bowel wall, one part being thin and relatively blownout and the next segment thickened. But there is no doubt about it, I am told that 'thumb-printing' is a very accurate sign.

QUESTION. May I ask how transient is the abdominal pain and rectal bleeding is your ischaemic cases. May it be as short as $24 \mathrm{hr}$ ?
Morson. Well, I'm a pathologist and all my clinical knowledge is second-hand.

QUESTION. The reason I asked this was that I recall seeing a young man of about 25 a month ago who complained of transient abdominal pain lasting for only a few hours, 2 or $3 \mathrm{hr}$ at the most, coupled with rectal bleeding, confirmed by intra-rectal examination which completely passed within the space of $24 \mathrm{hr}$. There was no abnormality on the barium enema or evidence of other disease.

Morson. The age here makes ischaemic colitis pretty unlikely. I wouldn't like to think that abdominal pain and rectal bleeding was exclusive to this disease.

WATKINSON. One of the radiological signs of systemic sclerosis that has been recognized for many years is this excessive sacculation of the colon. Is this also a vascular lesion?

Morson. I don't know how to resolve this difficulty, radiologically, but it's quite true what you say. Under the microscope in systemic sclerosis of the bowel you see this incredibly atrophied muscularis propria which is replaced by fibrous tissue, again a characteristic appearance. In this particular specimen I looked hard and there is nothing like this at all-quite the contrary, the muscle everywhere was very normal, it was thinned where it was sacculated but there was no fibrosis. We had other features, formation of granulation tissue which I personally have not seen in these surgical specimens in progressive systemic sclerosis of the bowel. But I would like to separate the two disorders.

Watkinson. One other question, how often have your clinical colleagues seen a pale cyanotic mucous membrane on sigmoidoscopy which became normal a few days later? I think I have seen this in a 70-year-old who had abdominal pain and rectal bleeding. I was tremendously impressed by the pallor and cyanosis in the mucosa.

Chatrman. Which mucosa?

WATKINSON. The sigmoid colon, and a few days later it was quite normal.

MoRson. I couldn't answer this of course, I don't know if anybody else here has any experience of this. You see Adrian Marston is very much against the idea, on anatomical grounds, that you can get ischaemic lesions of the rectum. You've got such a very good collateral blood supply.

Chatrman. I am sure Dr Morson would agree with me that this is a segmental lesion. If the rectum is diseased this eliminates the possibility of a vascular cause almost entirely. I presume that in the past this condition, which we can imagine was in existence before 3 or 4 years ago, produced funny segmental form of colitis that were called 'query segmental colitis' or Crohn's disease.

Morson. Possibly Dr Watkinson also brings to mind, that I have seen two examples of segmental colitis due to polyarteritis nodosa. This was of course a diagnosis made on a surgical specimen. Now, I am told that in the literature it says that in polyarteritis nodosa you get a curious sigoidoscope appearance with a sort of dusky cyanotic appearance. 